\title{
On Non-Equilibrium Dynamics of Multidimensional Infinite Particle Systems in the Translation Invariant Case
}

\author{
Ra. Siegmund-Schultze \\ Institute of Mathematics, Academy of Sciences, DDR-1086 Berlin, \\ German Democratic Republic
}

\begin{abstract}
It is shown that for a set of full measure with respect to any translation invariant probability distribution on the space of initial configurarations of classical particle systems on $\mathbb{R}^{d}$ with interaction given by a smooth superstable potential of finite range there is a solution to the Newtonian equations of motion, provided that the specific energy and the particle density of the initial configuration exist a.s.
\end{abstract}

\section{Introduction}

The infinite particle system approach to statistical mechanics raises the question of the existence of Newtonian dynamics for countable systems of interacting particles in Euclidean space $\mathbb{R}^{d}$. This means the following (if we confine ourselves to the case of pairwise interaction): Let be given a potential function $U: \mathbb{R}^{d} \curvearrowright \mathbb{R}$ fulfilling some regularity properties including smoothness and decay conditions. We consider the following infinite system of ordinary differential equations, concerning a sequence $\left\{x_{i}\right\}, i=1,2, \ldots$ of twice continuously differentiable functions $x_{i}:[0, \infty) \curvearrowright \mathbb{R}^{d}$ :

$$
\ddot{x}_{i}=-\sum_{j \neq i} \nabla U\left(x_{i}-x_{j}\right), \quad i=1,2, \ldots .
$$

The problem is to prove for a largest possible class of initial conditions

$$
\Phi_{0}=\left\{\left[x_{i}, v_{i}\right]\right\}_{i=1,2 \ldots}
$$

[where $\left.x_{i}=x_{i}(0), v_{i}=\dot{x}(0)\right]$ that a solution to $(N)$ exists. Naturally, we suppose that the initial configuration is locally finite, i.e. $\left\{x_{i}\right\}_{i=1,2, \ldots}$ has no accumulation points.

This is a purely analytical question. It is well-known that in the case of dimension one and two for some classes of potential functions $U$ Lanford [1] $(d=1)$, Dobrushin and Fritz $[3,4](d=1,2)$, and Gurevich and Suhov [15] $(d=1)$ succeeded to show the existence and (in a certain sense) the uniqueness of a solution to $(N)$ for large classes of initial configurations which are characterized by explicit conditions. 
For the purpose of equilibrium statistical mechanics it would be satisfying to prove the existence of dynamics [i.e. of solutions to $(N)$ ] for a set of full measure with respect to the equilibrium Gibbs states which correspond to the potential function $U$. Such results were obtained by Sinai [5,6], Marchioro et al. [7], Alexander [8], Lanford [2] for any dimension $d \geqq 1$.

This paper deals with the non-equilibrium dynamics in the case of random translation invariant initial conditions. The main result is of the same type as those concerning the equilibrium case. It can be shown that, for a set of full measure with respect to any translation invariant probability distribution on the space of initial configurations with respect to which specific energy and particle density are finite, one can find a solution to $(N)$, provided that the interaction potential $U$ has the following properties:

U1) $U$ is a $C^{2}$-function,

U2) $U(x)=U(-x)$ (Symmetry),

U3) $U$ has a bounded support, i.e. there is some $R>0$ such that $U(x)=0$ for $x \in \mathbb{R}^{d},|x| \geqq R$,

U4) $U$ is superstable, i.e. there are positive constants $a, b$ such that for each finite sequence $\left\{x_{1}, \ldots, x_{N}\right\}$ of elements of $\mathbb{R}^{d}$ we have

$$
\sum_{i \neq j} U\left(x_{i}-x_{j}\right) \geqq-a N+b \Pi,
$$

where $\Pi$ is the number of pairs $(i, j)$ such that $\left|x_{i}-x_{j}\right|<R$.

We get the existence of non-equilibrium dynamics for any dimension including the physically realistic case $d=3$.

The problem of uniqueness is still open. Especially it would be desirable to get a result that the limit procedure defined here leads to a unique solution for almost all initial configurations and that in that way there can be constructed a transformation group acting on a subset of full measure of the space of initial configurations.

We give a brief heuristic outline of the proof of the main result. A usual approach to infinite particle dynamics is, roughly speaking, to consider large boxes and the particles within those boxes, and then to enlarge the boxes to cover the whole space (and all particles). Then one has to prove the convergence of the trajectories of the finite particle systems towards a solution of $(N)$ for the whole system. We modify this approach a little in order to be able to make use of the translation invariance of (random) initial configurations. Instead of considering the finite subsystem of $\Phi_{0}$ lying in a large cube we consider the infinite periodical system which we obtain if we dissect the whole $\mathbb{R}^{d}$ into large cubic cells and endow each of them with the same finite subsystem of $\Phi_{0}$. Finally we shift the periodic system with a random vector being uniformly distributed over a cell, such that we get a random periodical and (statistically) translation invariant particle system. For periodic systems the existence of dynamics is obvious, since we may consider them as finite systems on a suitable torus. Then we show that, if we enlarge the cells to infinity, the sequence of translation invariant probability measures governing the bundle of trajectories of the periodical systems (of course, all randomness stems from the initial condition) is relatively compact, such that we can find a limit point in the sense of the weak topology. This limit point is a probability measure concentrated on solutions of $(N)$, where the initial values are equal to $\Phi_{0}$ in distribution. 
As in the deterministic case $[1,3,4]$ the main problem for the feasibility of this programme is to find an energy bound ensuring that there is no accumulation of large amounts of energy near the origin. In the deterministic case this is quite difficult (and unsolved for $d \geqq 3$ up to now). In the translation invariant random case considered here all things are easier, since now the origin is a place "as any other," a "typical place," where the energy is statistically distributed, and, in view of the conservation law for the specific energy being valid in the translation invariant case, cannot become too large.

Some Notations. Point process notations are taken from [10]. Counting measures are usually denoted by Greek capitals as $\Phi, \Psi$ (except for counting measures on function spaces). By $\delta_{x}$ we denote the Dirac measure concentrated on $x$. We denote by $\mu$ the Lebesgue measure on $\mathbb{R}^{d}$. We will use the notations $B, \operatorname{cl} B, \partial B$ for interior, closure, and boundary of a set $B$. The indicator function of a set $B$ is denoted by $\mathbb{1}_{B}$. The sets of integers and nonnegative integers are denoted by $\mathbb{Z}$ and $\mathbb{N}$, respectively. We introduce the following notations:

$$
\begin{aligned}
B_{s}(x) & :=\left\{y \in \mathbb{R}^{d}:|x-y| \leqq s\right\}, \\
B_{s} & :=B_{s}(0), \\
W_{s}(x): & =\left\{y \in \mathbb{R}^{d}: x_{i}-s / 2 \leqq y_{i}<x_{i}+s / 2\right\}, \\
W_{s} & :=W_{s}(0),
\end{aligned}
$$

for $s \in(0, \infty), x \in \mathbb{R}^{d}$. Let be $\ell \in \mathbb{N}^{d}$. Then we denote by $\|\ell\|$ the maximal coordinate of $\ell$.

\section{Newtonian Dynamics with Periodical Initial Conditions}

We consider the set $\mathbb{R}^{d} \times \mathbb{R}^{d}$ endowed with the following metric:

$$
d([x, v],[y, w]):=|x-y|+|v-w| /(1+|v-w|), \quad x, y, v, w \in \mathbb{R}^{d} .
$$

A counting measure is a measure $\Phi$ on $\left[\mathbb{R}^{d} \times \mathbb{R}^{d}, \Re^{d} \otimes \Re^{d}\right]$, which has the property that $\Phi(B) \in \mathbb{N}$ for any $d$-bounded set $B$ in $\Re^{d} \otimes \mathfrak{R}^{d}$. The set of all counting measures we denote by M. Let $\Phi$ be an element of M. Then (cf. [10, Proposition 1.2.1]) $\Phi$ has a representation

$$
\Phi=\sum_{1 \leqq i<I} \delta_{\left[x_{i}, v_{i}\right]}, \quad I \leqq \infty,
$$

as a sum of Dirac measures, where (in view of our choice of the metric $d$ ) $\left\{x_{i}\right\}_{i=1}^{I}$ has no accumulation points. So we may interpret $\Phi$ as particle configuration (with given positions $x_{i}$ and velocities $v_{i}$ ).

In the following we will denote by $\Phi^{\times}$, for a counting measure

$$
\Phi=\sum_{1 \leqq i<I} \delta_{\left[x_{i}, v_{i}\right]} \text {, }
$$

the measure

$$
\Phi^{\times}:=\sum_{1 \leqq i<I} \delta_{x_{i}}
$$


which is obtained from $\Phi$ by cancelling all velocity "marks" (such that $\Phi^{\times}$is a counting measure on $\mathbb{R}^{d}$ ).

Let, for each $y \in \mathbb{R}^{d}, T_{y}$ denote the spatial shift operator on $\mathbb{R}^{d} \times \mathbb{R}^{d}$ defined by $T_{y}[x, v]:=[x+y, v]$ which extends to $\mathrm{M}$ by the set-up

$$
T_{y} \Phi(X):=\Phi\left(T_{y} X\right), \quad X \in \mathbb{R}^{d} \otimes \mathbb{R}^{d},
$$

where

$$
T_{y} X:=\left\{[x, v] \in \mathbb{R}^{d} \times \mathbb{R}^{d}:[x-y, v] \in X\right\} .
$$

Let $F_{c}$ denote the set of all continuous bounded real functions $f$ on $\mathbb{R}^{d} \times \mathbb{R}^{d}$ having a $d$-bounded support, i.e. $f(x, v)=0$ for $|x| \geqq C_{f}, C_{f}$ being some real number depending on $f$. The vague topology on $\mathrm{M}$ is defined as the weakest topology making all mappings

$$
\Phi \in M \frown \sum_{i \in I(\Phi)} f\left(x_{i}, v_{i}\right) \in \mathbb{R}, \quad f \in F_{c}
$$

continuous. Here

$$
\sum_{i \in I(\Phi)} f\left(x_{i}, v_{i}\right):=\sum_{1 \leqq i<I} f\left(x_{i}, v_{i}\right)
$$

for

$$
\Phi=\sum_{1 \leqq i<I} \delta_{\left[x_{i}, v_{i}\right]}
$$

It is well-known that the vague topology makes $M$ a Polish space (cf. [10, Chap. 1.9]). The corresponding $\sigma$-field of Borel sets we denote by $\mathfrak{M}$.

We want to give without proof (see [14]) a simple characterization of the vague convergence of counting measures in terms of their atoms, which is valid not only in the context of our special phase space $\mathbb{R}^{d} \times \mathbb{R}^{d}$, but also for counting measures on arbitrary complete separable metric spaces $A$. Let be given a sequence $\left\{\Phi_{k}\right\}_{k=0,1,2, \ldots}$ of elements of M. Then the vague convergence $\Phi_{k} \underset{k \rightarrow \infty}{\longrightarrow} \Phi_{0}$ takes place iff there are representations (for some $I \leqq \infty)$

$$
\begin{gathered}
\Phi_{0}=\sum_{1 \leqq i<I} \delta_{a_{i}}, \\
\Phi_{k}=\sum_{1 \leqq i<N(k)} \delta_{a_{i, k}}+\chi_{k}, \quad k=1,2, \ldots
\end{gathered}
$$

such that the following relations hold:

Ai) $N(k) \uparrow I$ as $k \rightarrow \infty$,

Aii) $a_{i, k} \rightarrow a_{i}$ as $k \rightarrow \infty$,

Aiii) For each bound set $B \subseteq A$ there exists a $k^{\prime} \in \mathbb{N}$ such that

$$
\left(\sum_{k^{\prime} \leqq i<N(k)} \delta_{a_{i, k}}+\chi_{k}\right)(B) \underset{k \rightarrow \infty}{\longrightarrow} 0 .
$$

A probability distribution $P$ on $[\mathrm{M}, \mathfrak{M}]$ will be called translation invariant, if for any $y \in \mathbb{R}^{d}$ we have $P=P \circ T_{y}^{-1}$. We call $\Phi$ a $b$-periodic configuration, for $b \in(0, \infty)$, if $T_{b y} \Phi=\Phi$ for each $y \in \mathbb{Z}^{d}$. Let $\mathbf{M}_{b}$ denote the set of all $b$-periodic counting measures. 
Consider a $\Phi_{0} \in \mathrm{M}_{b}, \Phi_{0} \neq 0$, and choose a representation

$$
\Phi_{0}=\sum_{i=1}^{\infty} \delta_{\left[x_{i}, v_{i}\right]} .
$$

It is an immediate consequence of the existence and uniqueness theorem for ordinary differential equations on smooth manifolds (see [12, Chap. 5.3]) that there is a unique solution $\left\{x_{i}\right\}_{i=1}^{\infty}$ of $(N)$ with initial condition $\left\{\left[x_{i}, v_{i}\right]\right\}_{i=1}^{\infty}$, such that

$$
\Phi_{t}:=\sum_{i=1}^{\infty} \delta_{\left[x_{t}(t), \dot{x}_{t}(t)\right]} \in \mathrm{M}_{b} \text { for all } t \geqq 0 .
$$

Indeed, there is a one-to-one correspondence between counting measures in $\mathrm{M}_{b}$ having the property $\Phi^{\times}\left(W_{b}\right)=n$, and points of the manifold $\mathbb{T}^{d n} \times \mathbb{R}^{d n}$, where $\mathbb{T}^{d n}$ is the $d n$-dimensional torus obtained from $\left(W_{b}\right)^{n}$ by an identification of opposite sides. In view of the finiteness of the interaction radius $R$ we may consider $(N)$ as an ordinary first order differential equation on $\mathbb{T}^{d n} \times \mathbb{R}^{d n}$, where the existence of the solution for all times $t \geqq 0$ follows in the usual way from the energy conservation law, which ensures the boundedness of the velocities.

Obviously the (arbitrary) way of indexing $\Phi_{0}$ does not influence $\Phi_{t}$, so that $(N)$ defines a family of mappings

$$
S_{t}^{b}: \mathrm{M}_{b} \curvearrowright \mathrm{M}_{b}, \quad t \geqq 0 .
$$

Using the vague convergence criterion given above it is easy to see that the one-toone correspondence between

$$
\mathrm{M}_{b}^{n}:=\left\{\Phi \in \mathrm{M}_{b}: \Phi^{\times}\left(W_{b}\right)=n\right\}
$$

and $\mathbb{T}^{d n} \times \mathbb{R}^{d n}$ is a homeomorphism with respect to the vague topology in $\mathbf{M}_{b}^{n}$ (i.e. the restriction of the vague topology in M). This fact together with the theorem about continuous dependence of solutions of ordinary differential equations on initial values implies

Lemma 1. $S_{t}^{b}$ is, for any $t \geqq 0$, a vaguely continuous mapping from $\mathbf{M}_{b}$ into itself.

It is also clear that $S_{t}^{b}$ is translation invariant, i.e.

$$
T_{y} \circ S_{t}^{b}=S_{t}^{b} \circ T_{y} \text { for any } y \in \mathbb{R}^{d}, \quad t \geqq 0 .
$$

Fix some $b>1$ and consider the following mapping

$$
p_{b}: \Phi=\sum_{1 \leqq i<I} \delta_{\left[x_{i}, v_{i}\right]} \in \mathrm{M} \curvearrowright p_{b}(\Phi):=\sum_{1 \leqq i<I} \mathbb{1}_{W_{b}}\left(x_{i}\right) \sum_{z \in \mathbb{Z}^{d}} \delta_{\left[x_{i}+b z, v_{i}\right]} \in \mathrm{M}_{b} .
$$

It is easy to derive from the definition of $\mathfrak{M}$ (see [10, Chap. 1]), that $p_{b}$ is a $\mathfrak{M}$-measurable mapping from $M$ into itself and that $M_{b}$ is an element of $\mathfrak{M}$. We set $\mathfrak{M}_{b}:=\mathrm{M}_{b} \cap \mathfrak{M}$. So $p_{b}$ transforms any probability distribution $P$ on $[\mathrm{M}, \mathfrak{M}]$ to a probability distribution $\widetilde{P}^{b}:=P \circ p_{b}^{-1}$ on $[\mathrm{M}, \mathfrak{M}]$ which is concentrated on the measurable subspace $\left[\mathrm{M}_{b}, \mathfrak{M}_{b}\right]$. Now we consider the following mixture:

$$
P^{b}:=\frac{1}{b^{d}} \int_{W_{b}} \tilde{P}^{b} \circ T_{x}^{-1} \mu(d x) .
$$

Clearly we have 
Lemma 2. $P^{b}$ is translation invariant.

We assume from now on that $P$ is translation invariant itself.

Lemma 3. For $b \rightarrow \infty$ we have the weak convergence relation $P^{b} \Rightarrow P$.

Proof. Let $X$ be a $d$-bounded subset of $\mathbb{R}^{d} \times \mathbb{R}^{d}$ and let $Y$ be an element of $\mathfrak{M}$. We have

$$
\begin{aligned}
P^{b}(\Phi(* \cap X) \in Y)= & b^{-d} \int_{W_{b}} \mu(d x) \tilde{P}^{b}\left(\left(T_{x} \Phi\right)(* \cap X) \in Y\right) \\
= & b^{-d} \int_{W_{b}} \mu(d x) \tilde{P}^{b}\left(\Phi\left(* \cap T_{x} X\right) \in T_{-x} Y\right) \\
= & b^{-d} \int_{\left\{x \in W_{b}: T_{x} X \leqq W_{b} \times \mathbb{R}^{d}\right\}} \mu(d x) \tilde{P}^{b}\left(\Phi\left(* \cap T_{x} X\right) \in T_{-x} Y\right) \\
& +b^{-d} \int_{\left\{x \in W_{b}: T_{x} X_{\Phi} W_{b} \times \mathbb{R}^{d}\right\}} \mu(d x) \tilde{P}^{b}\left(\Phi\left(* \cap T_{x} X\right) \in T_{-x} Y\right) \\
= & b^{-d} \int_{\left\{x \in W_{b}: T_{x} X \cong W_{b} \times \mathbb{R}^{d}\right\}} \mu(d x) P\left(p_{b}(\Phi)\left(* \cap T_{x} X\right) \in T_{-x} Y\right)+o(1) \\
= & b^{-d} \int_{W_{b}} \mu(d x) P\left(\left(T_{x} \Phi\right)(* \cap X) \in Y\right)+o(1) \\
= & b^{-d} \int_{W_{b}} \mu(d x) P(\Phi(* \cap X) \in Y)+o(1) \\
= & P(\Phi(* \cap X) \in Y)+o(1), \quad b \rightarrow \infty,
\end{aligned}
$$

such that the desired weak convergence holds for the restriction of the particle system to bounded sets. This implies the statement of the lemma (cf. [10, Proposition 4.1.12]).

In view of Lemma 1 we can define probability distributions

$$
P_{t}^{b}:=P^{b} \circ\left(S_{t}^{b}\right)^{-1} \text {. }
$$

Since $S_{0}^{b}=\mathrm{id}_{\mathrm{M}_{b}}$, we have $P_{0}^{b}=P^{b}$.

\section{Specific Energy}

Let $\Phi$ be an element of $\mathrm{M}$ and let $\left[x_{i}, v_{i}\right]$ denote the position and velocity of a particle in $\Phi$. By the energy of this particle we understand the (finite) expression

$$
\frac{1}{2}\left|v_{i}\right|^{2}+\frac{1}{2} \sum_{\substack{j \in I(\Phi) \\ j \neq i}} U\left(x_{i}-x_{j}\right)=: e(\Phi, i) .
$$

Consider the energy of $\Phi$ within the unit cube

$$
e\left(\Phi, W_{1}\right):=\sum_{i \in I(\Phi)} \mathbb{1}_{W_{1}}\left(x_{i}\right) e(\Phi, i),
$$

which is a measurable function of $\Phi$.

Let $P$ be a translation invariant probability measure which satisfies the following two conditions:

1) $\bar{m}:=\mathbb{E}_{p} \Phi^{\times}\left(W_{1}\right)<\infty$,

2) $\bar{e}:=\mathbb{E}_{P} e\left(\Phi, W_{1}\right)$ exists

i.e. $P$ is supposed to have finite mean particle density and specific energy. 
Taking into account the superstability of the potential $U$ we have the following Lemma 4. There is a finite constant $C=C(\bar{m}, \bar{e})>0$ (depending on $P$ only through $\bar{m}$ and $\bar{e})$ such that

$$
\bar{q}:=\mathbb{E}_{P}\left[\Phi^{\times}\left(W_{1}\right)\right]^{2} \leqq C .
$$

Proof. 1. We will show that $\bar{q}$ is finite. For any $N \in \mathbb{N}$ consider the cube $W_{2 N R}$ which can be cut into smaller cubes of edge length $2 R$,

$$
W_{2 N R}=\bigcup_{\substack{\left(\ell_{1}, \ldots, \ell_{d}\right) \in \mathbb{N}^{d} \\\|\ell\| \leqq N-1}} W_{2 R}(x(N)+2 R \ell),
$$

where $x(N):=[-(N-1) R, \ldots,-(N-1) R] \in \mathbb{R}^{d}$. Now we have for any $\Phi \in \mathrm{M}$

$$
e\left(\Phi, W_{2 N R}\right)=e_{\mathrm{kin}}+e_{\mathrm{int}}+e_{b d},
$$

where

$$
\begin{aligned}
e_{\mathrm{kin}}: & =\frac{1}{2} \sum_{i \in I(\Phi)}\left|v_{i}\right|^{2} \mathbb{1}_{W_{2 N R}}\left(x_{i}\right), \\
e_{\mathrm{int}} & :=\frac{1}{2} \sum_{\substack{i, j \in I(\Phi) \\
i \neq j}} U\left(x_{i}-x_{j}\right) \mathbb{1}_{W_{2 N R}}\left(x_{i}\right) \mathbb{1}_{W_{2 N R}}\left(x_{j}\right), \\
e_{b d} & :=\frac{1}{2} \sum_{i, j \in I(\Phi)} U\left(x_{i}-x_{j}\right) \mathbb{1}_{W_{2 N R}}\left(x_{i}\right)\left[1-\mathbb{1}_{W_{2 N R}}\left(x_{j}\right)\right] .
\end{aligned}
$$

In view of the superstability of $U$ we have the estimate

$$
e\left(\Phi, W_{2 N R}\right) \geqq-a \Phi^{\times}\left(W_{2 N R}\right)+b \Pi\left(\Phi, W_{2 N R}\right)+e_{b d},
$$

where for any Borel set $B$ we denote by $\Pi(\Phi, B)$ the number of pairs $(i, j)$ such that $x_{i}, x_{j} \in B$ and $\left|x_{i}-x_{j}\right|<R$. Then we get

$$
e\left(\Phi, W_{2 N R}\right) \geqq-a \Phi^{\times}\left(W_{2 N R}\right)+b \sum_{\substack{\ell \in \mathbb{N}^{d} \\\|\ell\| \leqq N-1}} \Pi\left(\Phi, W_{2 R}(x(N)+2 R \ell)\right)+e_{b d} .
$$

Obviously there exist some absolute constants $\alpha, \beta>0$ such that for any $\Phi \in M$, we have $\Pi\left(\Phi, W_{2 R}\right) \geqq \alpha\left[\Phi^{\times}\left(W_{2 R}\right)\right]^{2}-\beta$. So

$$
\begin{aligned}
e\left(\Phi, W_{2 N R}\right) \geqq & -a \Phi^{\times}\left(W_{2 N R}\right) \\
& +b \alpha \sum_{\substack{\ell \in \mathbb{N}^{d} \\
\|\ell\| \leqq N-1}}\left[\Phi^{\times}\left(W_{2 R}(x(N)+2 R \ell)\right)\right]^{2}-b \beta N^{d}+e_{b d} .
\end{aligned}
$$

Let us denote by $\bar{u}$ the supremum of $|U|+1$. Then we get

$$
\left|e_{b d}\right| \leqq \bar{u} \Pi\left(\Phi, W_{(2 N+2) R} \backslash W_{(2 N-2) R}\right) .
$$

Any pair of particles in $\Phi$ with $\left|x_{i}-x_{j}\right|<R$ and

$$
x_{i}, x_{j} \in W_{(2 N+2) R} \backslash W_{(2 N-2) R}
$$

is covered by some cube $W_{2 R}\left(x_{0}(N)+R \ell\right)$, where $x_{0}(N)$ is the vector $(-N R, \ldots,-N R) \in \mathbb{R}^{d}$ and $\ell \in \mathbb{N}^{d},\|\ell\| \leqq 2 N$, and at least one coordinate of $\ell$ is 0 or $2 N$. We denote the set of such $\ell$ by $L(N)$. Then we have

$$
\left|e_{b d}\right| \leqq \bar{u} \sum_{\ell \in L(N)}\left[\Phi^{\times}\left(W_{2 R}\left(x_{0}(N)+R \ell\right)\right)\right]^{2} .
$$


This together with (2.2) yields

$$
\begin{aligned}
e\left(\Phi, W_{2 N R}\right) \geqq & -a \Phi^{\times}\left(W_{2 N R}\right)-b \beta N^{d} \\
& +b \alpha \sum_{\substack{\ell \in N^{d} \\
\|\ell\| N}}\left[\Phi^{\times}\left(W_{2 R}(x(N)+2 R \ell)\right)\right]^{2} \\
& -\bar{u} \sum_{\ell \in L(N)}\left[\Phi^{\times}\left(W_{2 R}\left(x_{0}(N)+R \ell\right)\right)\right]^{2} .
\end{aligned}
$$

We introduce the following notation (for any $\Phi \in \mathbf{M}, x \in \mathbb{R}^{d}, 1 \leqq k \leqq d$ ),

$$
\gamma_{N}(\Phi, x, k):=\sum_{\ell \in L_{k}(N)}\left[\Phi^{\times}\left(W_{2 R}(x+2 R \ell)\right)\right]^{2},
$$

where $L_{k}(N)$ is the set of all $\ell \in \mathbb{N}^{d}$ with $\|\ell\| \leqq N-1$ and $\ell_{k}=0$.

Let $e_{k}$ be the unit vector in direction of the $k^{\text {th }}$ coordinate axis. Now from the relation (2.3) we get

$$
\begin{aligned}
e(\Phi, & \left.W_{2 N R}\right)+a \Phi^{\times}\left(W_{2 N R}\right)+b \beta N^{d} \\
& +\bar{u} \sum_{k=1}^{d} \sum_{i=0}^{1} \sum_{y \in E_{d}} \gamma_{N}\left(\Phi, x_{0}(N)+i 2 N R e_{k}+y R, k\right) \\
\geqq & \frac{b \alpha}{d} \sum_{k=1}^{d} \sum_{m=0}^{N-1} \gamma_{N}\left(\Phi, x(N)+2 R m e_{k}, k\right) .
\end{aligned}
$$

Here $E_{d}$ is the set of vectors with all coordinates being 0,1 or 2 . For $\sigma>0$ we define a continuous function $f_{\sigma}:[0, \infty) \curvearrowright[0, \infty)$ by means of the set-up

$$
f_{\sigma}(s):=\left\{\begin{array}{lll}
s & \text { for } & s \leqq \sigma \\
\sqrt{\sigma s} & \text { for } & s>\sigma .
\end{array}\right.
$$

For each $\sigma>0$ the function $f_{\sigma}$ has the following properties:

i) $f_{\sigma}$ is concave,

ii) $f_{\sigma}\left(s+s^{\prime}\right) \leqq f_{\sigma}(s)+f_{\sigma}\left(s^{\prime}\right), s, s^{\prime} \geqq 0$,

iii) $\lambda f_{\sigma}(s) \geqq f_{\sigma}(\lambda s) \geqq \sqrt{\lambda} f_{\sigma}(s), s \geqq 0, \lambda \geqq 1$,

iv) $f_{\sigma}(s) \leqq s, s \geqq 0$.

So we get (using Jensen's inequality) for any $\sigma>0, N \geqq(b \alpha)^{-1}$ :

$$
\begin{aligned}
\mid e(\Phi, & \left.W_{2 N R}\right) \mid+a \Phi^{\times}\left(W_{2 N R}\right)+b \beta N^{d} \\
& +\bar{u} \sum_{k=1}^{d} \sum_{i=0}^{1} \sum_{y \in E_{d}} f_{\sigma}\left(\gamma_{N}\left(\Phi, x_{0}(N)+i 2 N R e_{k}+y R, k\right)\right) \\
\geqq & d^{-1} \sqrt{b \alpha N^{-1}} \sum_{k=1}^{d} \sum_{m=0}^{N-1} f_{\sigma}\left(\gamma_{N}\left(\Phi, x(N)+2 R m e_{k}, k\right)\right) .
\end{aligned}
$$

From the definition of $\gamma_{N}$ and the property $\bar{m}<\infty$, it follows that

$$
g(\sigma, N, k):=\mathbb{E} f_{\sigma}\left(\gamma_{N}(\Phi, x, k)\right)<\infty,
$$

where the fact that this expectation does not depend on $x$ is a consequence of the translation invariance of $P$. 
So from (2.5) we deduce

$$
\begin{aligned}
& \mathbb{E}\left|e\left(\Phi, W_{2 N R}\right)\right|+a \bar{m}(2 N R)^{d}+b \beta N^{d}+2 \bar{u} \cdot 3^{d} \sum_{k==1}^{d} g(\sigma, N, k) \\
& \geqq d^{-1} \sqrt{b \alpha N} \sum_{k=1}^{d} g(\sigma, N, k),
\end{aligned}
$$

such that

$$
\left[d^{-1} \sqrt{b \alpha N}-3^{d} \cdot 2 \bar{u}\right] \sum_{k=1}^{d} g(\sigma, N, k) \leqq \mathbb{E}\left|e\left(\Phi, W_{2 N R}\right)\right|+\left(a \bar{m}(2 R)^{d}+b \beta\right) N^{d} .
$$

For sufficiently large $N$ the factor in square brackets on the left-hand side is positive. With $\sigma \rightarrow \infty$, we get that

$$
\mathbb{E} \gamma_{N}(\Phi, x, k)<\infty, \quad x \in \mathbb{R}^{d}, \quad k=1,2, \ldots, d .
$$

Hence we have

$$
\mathbb{E}\left[\Phi^{\times}\left(W_{2 R}\right)\right]^{2}<\infty .
$$

2. Now in (2.3) we may form expectations on each side: So we have

$$
(2 R)^{d} \bar{e}+\left(a(2 R)^{d} \bar{m}+b \beta\right) \geqq\left[b \alpha-2 \bar{u} d N^{-1}\left(2+N^{-1}\right)^{d-1}\right] \mathbb{E}\left[\Phi^{\times}\left(W_{2 R}\right)\right]^{2} .
$$

In that way we get for sufficiently large $N$ an upper bound for $\mathbb{E}\left[\Phi^{\times}\left(W_{2 R}\right)\right]^{2}$ depending only (linearly) on $\bar{e}$ and $\bar{m}$. Now we choose an integer $k_{0}$ such that $2 k_{0} R \geqq 1$. Then we have

$$
\mathbb{E}\left[\Phi^{\times}\left(W_{1}\right)\right]^{2} \leqq \mathbb{E}\left[\Phi^{\times}\left(W_{2 k_{0} R}\right)\right]^{2} .
$$

Cutting the cube $W_{2 k_{0} R}$ into $\left(k_{0}\right)^{d}$ cubes of edge length $2 R$ we get, making use of the translation invariance of $P$ and of the Schwarz inequality

$$
\bar{q}=\mathbb{E}\left[\Phi^{\times}\left(W_{1}\right)\right]^{2} \leqq k_{0}^{2 d} \mathbb{E}\left[\Phi^{\times}\left(W_{2 R}\right)\right]^{2} \leqq C(\bar{m}, \bar{e}) .
$$

Let us fix some translation invariant probability measure $P$ having finite specific energy $\bar{e}$ and particle density $\bar{m}$. Let $b>1$ be given. It is easy to derive from the definition of $P^{b}$ that this probability measure has a finite second moment $\bar{q}^{b}:=\mathbb{E}_{p b}\left[\Phi^{\times}\left(W_{1}\right)\right]^{2}$, too, and that $\bar{q}^{b} \leqq C^{\prime} \bar{q}$ for some absolute constant $C^{\prime}$ (we can choose $C^{\prime}=2^{2 d}$ ).

From this we derive that the absolute value $\left|\bar{e}^{-b}\right|$ of the specific energy is bounded by a constant independent of $b$ :

$$
\left|\bar{e}^{b}\right| \leqq C \text {. }
$$

It is clear that the mean particle density $m$ remains unchanged when passing from $P$ to $P^{b}$.

Let us investigate what happens under time evolution. From the fact that $S_{t}^{b}$ leaves the particle number in $W_{b}$ invariant (this is in fact the number of particles of the finite torus system considered above) we easily derive that

$$
\bar{m}_{t}^{b}:=\mathbb{E}_{P_{t}} \Phi^{\times}\left(W_{1}\right) \equiv \bar{m}, \quad t \geqq 0,
$$


if we take into account the translation invariance of $P^{b}$. On the other hand it is well-known that the energy of the finite torus system is an invariant of the motion. Since this energy is equal to $e\left(\Phi, W_{b}\right)$ for $\Phi \in \mathrm{M}_{b}$, we may conclude that $S_{t}^{b}$ leaves the specific energy unchanged, too. So

$$
\bar{e}_{t}^{b} \equiv \bar{e}^{b}, \quad t \geqq 0 .
$$

This together with Lemma 4 leads to the conclusion that there is a constant $C_{0}$ (not depending on $b$ ) such that

$$
\bar{q}_{t}^{b} \leqq C_{0}
$$

for all $t \geqq 0$.

\section{Energy Bounds}

Let us consider a sequence $V_{0}, V_{1}, \ldots$ of continuously differentiable functions on $\mathbb{R}^{d}$ with finite support having the properties

$$
\begin{array}{lll}
V_{k}(x)=1 & \text { for } & |x| \leqq k+R, \\
0 \leqq V_{k}(x) \leqq 1 & \text { for } & |x| \leqq k+R+1, \\
V_{k}(x)=0 & \text { for } & |x|>k+R+1, \\
\left|\nabla V_{k}(x)\right| \leqq 2 & \text { for } & x \in \mathbb{R}^{d} .
\end{array}
$$

On the other hand we introduce a sequence $f_{0}, f_{1}, f_{2}, \ldots$ of continuously differentiable functions on $\mathbb{R}^{d}$ with finite support fulfilling the conditions

$$
\begin{array}{lll}
f_{k}(x)=1 & \text { for } & |x| \leqq k R+\frac{1}{2}(k+2)(k+3), \\
0 \leqq f_{k}(x) \leqq 1 & \text { for } & |x| \leqq k R+\frac{1}{2}(k+2)(k+3)+1, \\
f_{k}(x)=0 & \text { for } & |x|>k R+\frac{1}{2}(k+2)(k+3)+1, \\
\left|\nabla f_{k}(x)\right| \leqq 2 & \text { for } & x \in \mathbb{R}^{d} .
\end{array}
$$

Using these functions we define "modified energy functions"

$$
e_{k}(\Phi):=\sum_{i \in I(\Phi)} f_{k}\left(x_{i}\right)\left[\frac{1}{2}\left|v_{i}\right|^{2}+\frac{1}{2} \sum_{\substack{j \in I(\Phi) \\ j \neq i}} V_{k}\left(x_{i}-x_{j}\right)\right] .
$$

For any bounded Borel set $B \in \mathbb{R}^{d}$ we introduce the notation

$$
e_{0}(\Phi, B):=\sum_{i \in I(\Phi)}\left[\frac{1}{2}\left|v_{i}\right|^{2}+\frac{1}{2} \sum_{\substack{j \in I(\Phi) \\ j \neq i}} V_{0}\left(x_{i}-x_{j}\right)\right] \mathbb{1}_{B}\left(x_{i}\right) .
$$

Let $\Phi_{0} \in \mathrm{M}_{b}$. Then, writing $\Phi_{t}:=S_{t}^{b} \Phi_{0}$, we see that all functions $e_{k}\left(\Phi_{b}\right)$ are continuously differentiable with respect to $t$ and we get

$$
\begin{aligned}
\frac{d}{d t} e_{k}\left(\Phi_{t}\right)= & \sum_{i \in \Gamma(\Phi)}\left\{\nabla f_{k}\left(x_{i}(t)\right) \cdot \dot{x}_{i}(t)\left[\frac{1}{2}\left|\dot{x}_{i}(t)\right|^{2}+\sum_{j \neq i} V_{k}\left(x_{i}(t)-x_{j}(t)\right)\right]\right. \\
& +f_{k}\left(x_{i}(t)\right) \sum_{j \neq i}\left(-\dot{x}_{i}(t) \nabla U\left(x_{i}(t)-x_{j}(t)\right)\right. \\
& \left.+\frac{1}{2}\left(\dot{x}_{i}(t)-\dot{x}_{j}(t)\right) \cdot \nabla V_{k}\left(x_{i}(t)-x_{j}(t)\right)\right\} .
\end{aligned}
$$


Hence we have, using $|\nabla U| \leqq \overline{\bar{u}}$ for some $\overline{\bar{u}}$,

$$
\begin{aligned}
& \left|\frac{d}{d t} e_{k}\left(\Phi_{t}\right)\right| \leqq 4\left[e_{k+1}\left(\Phi_{t}\right)\right]^{3 / 2} \\
& \quad+\sum_{i \in I(\Phi)} f_{k}\left(x_{i}(t)\right)\left[\sum_{j \neq i} V_{k+1}\left(x_{i}(t)-x_{j}(t)\right)\left((\overline{\bar{u}}+1) \dot{x}_{i}(t)|+| \dot{x}_{j}(t) \mid\right)\right] \\
& \leqq \\
& \quad(2 \overline{\bar{u}}+8)\left[e_{k+1}\left(\Phi_{t}\right)\right]^{3 / 2} .
\end{aligned}
$$

Fix some $T \geqq 1$. Choose a constant $L \geqq 1$ and assume that, for some $k$,

$$
\sup _{0 \leqq t \leqq T} e_{k}\left(\Phi_{t}\right) \geqq 2^{k+1} L, \quad \sup _{0 \leqq t \leqq T} e_{k+1}\left(\Phi_{t}\right)<2^{k+2} .
$$

Let $t_{0}$ be chosen such that $e_{k}\left(\Phi_{t_{0}}\right) \geqq 2^{k+1} L$. Then we would have, in view of (3.1), $e_{k}\left(\Phi_{t}\right) \geqq 2^{k} L$ for any $t \in[0, T]$ with

$$
\begin{aligned}
\left|t-t_{0}\right| & \leqq\left(2^{k+1} L-2^{k} L\right)\left(\sup _{0 \leqq s \leqq T}\left|\frac{d}{d s} e_{k}\left(\Phi_{s}\right)\right|\right)^{-1} \\
& \leqq 2^{k} L(2 \overline{\bar{u}}+8)^{-1}\left(2^{k+2} L\right)^{-3 / 2} \\
& \leqq(2 \bar{u}+8)^{-1} L^{-1 / 2} 2^{-k / 2-3} .
\end{aligned}
$$

Now from $T, L \geqq 1$ it easily follows that $e_{k}\left(\Phi_{t}\right) \geqq 2^{k} L$ on a subset of $[0, T]$ of measure not less than $(2 \overline{\bar{u}}+8)^{-1} L^{-1 / 2} 2^{-k / 2-3}$, such that

$$
\int_{0}^{T} e_{k}\left(\Phi_{t}\right) d t \geqq(2 \overline{\bar{u}}+8)^{-1} L^{1 / 2} 2^{k / 2-3} .
$$

This leads to the conclusion that either

$$
\sup _{0 \leqq t \leqq T} e_{0}\left(\Phi_{t}\right)<2 L
$$

or

$$
\int_{0}^{T} e_{k}\left(\Phi_{t}\right) d t \geqq[8(2 \overline{\bar{u}}+8)]^{-1} L^{1 / 2} 2^{k / 2} \quad \text { for some } k
$$

or

$$
\sup _{0 \leqq t \leqq T} e_{k}\left(\Phi_{t}\right) \geqq 2^{k+1} L \quad \text { for every } k .
$$

1. Let us first discuss the third possibility. From the fact that the energy and the particle number in $W_{b}$ are invariants of $S_{t}^{b}$ (hence velocities are bounded for given $\Phi_{0} \in M_{b}$ ) we easily infer that (iii) is impossible.

2. Consider (ii). We get using the Fubini theorem

$$
\alpha_{k}:=\iint_{0}^{T} e_{k}\left(\Phi_{t}\right) d t P^{b}\left(d \Phi_{0}\right)=\iint_{0}^{T} \int e_{k}\left(\Phi_{t}\right) P^{b}\left(d \Phi_{0}\right) d t
$$

From the definition of $e_{k}$ we get for some $K, K^{\prime}, K^{\prime \prime}>0$ (not depending on $b$ )

$$
\begin{aligned}
\alpha_{k} \leqq & \int_{0}^{T} \int\left\{\left[e\left(\Phi, B_{K(k+1)^{2}}\right)+\bar{u}\left(\Phi^{\times}\left(B_{K(k+1)^{2}}\right)\right)^{2}\right]\right. \\
& \left.+\left(\Phi^{\times}\left(B_{K(k+1)^{2}}\right)\right)^{2}\right\} P_{t}^{b}(d \Phi) d t \\
\leqq & \int_{0}^{T}\left[K^{\prime}(k+1)^{2 d} \bar{e}_{t}^{b}+K^{\prime \prime}(k+1)^{4 d} \bar{q}_{t}^{b}\right] d t
\end{aligned}
$$


Here we estimated $\mathbb{E}_{P_{t}^{b}}\left[\Phi^{\times}\left(B_{K(k+1)^{2}}\right)\right]^{2}$ by $\bar{q}_{t}^{b}=\mathbb{E}_{P_{t}^{b}}\left[\Phi^{\times}\left(W_{1}\right)\right]^{2}$ in the way which was shown at the end of the proof of Lemma 4. So we get, using (2.6), (2.8), and (2.9)

$$
\alpha_{k} \leqq T K^{\prime \prime \prime}(k+1)^{4 d}
$$

for some $K^{\prime \prime \prime}$ not depending on $b$.

From this we derive, using the Markov inequality for some $K_{0}, K_{1}>0$,

$$
\begin{aligned}
& P^{b}\left(\bigcup_{k \geqq 0}\left\{\int_{0}^{T} e_{k}\left(\Phi_{t}\right) d t \geqq[8(2 \bar{u}+8)]^{-1} L^{1 / 2} 2^{k / 2}\right\}\right) \\
& \leqq L^{-1 / 2} T K_{0} \sum_{k \geqq 0} 2^{-k / 2}(k+1)^{4 d}=L^{-1 / 2} K_{1} T .
\end{aligned}
$$

From 1. and 2. we easily infer that, for any bounded Borel set $B \subset \mathbb{R}^{d}$,

\section{Lemma 5.}

$$
P^{b}\left(\sup _{0 \leqq t \leqq T} e_{0}\left(\Phi_{t}, B\right) \geqq L\right) \leqq K(B) T L^{-1 / 2}, \quad T, L \geqq 1,
$$

where the constant $K(B)$ does not depend on $b$.

\section{A Velocity Bound}

Let $\Phi$ be an element of $\mathrm{M}_{b}$. Denote by $x_{i}$ the trajectory of the $i^{\text {th }}$ particle with respect to $S_{t}^{b}$ (for any fixed indexing). We consider the following event (not depending on the indexing)

$$
\begin{aligned}
Y_{t, T}^{M, b}:= & \left\{\Phi \in \mathrm{M}_{b}: \text { There is an } i \in I(\Phi) \text { and a } \tau \in[0, T]\right. \\
& \text { such that } \left.x_{i}(t) \in W_{1},\left|\dot{x}_{i}(\tau)\right|>M\right\} .
\end{aligned}
$$

(From the theorem about continuous dependence of the solutions of ordinary differential equations on initial data, it can be derived that this is a measurable subset of M.)

Now we get, estimating the acceleration of the particles

$$
\begin{aligned}
P^{b}\left(Y_{t, T}^{M, b}\right) \leqq & P^{b}\left(\left\{\Phi \in \mathrm{M}_{b}: \sum_{i \in I(\Phi)}\left[\mathbb { 1 } _ { W _ { 1 } } ( x _ { i } ( t ) ) \left(\left|v_{i}\right|\right.\right.\right.\right. \\
& \left.\left.\left.\left.+\int_{0}^{T} \sum_{j \in I(\Phi)}\left|\nabla U\left(x_{j}(r)-x_{i}(r)\right)\right| d r\right)\right]>M\right\}\right) \\
\leqq & M^{-1} \int P^{b}(d \Phi)\left\{\sum_{i \in I(\Phi)} \mathbb{1}_{W_{1}}\left(x_{i}(t)\right)\left(\left|v_{i}\right|+\int_{0}^{T} \sum_{j \in I(\Phi)}\left|\nabla U\left(x_{j}(r)-x_{i}(r)\right)\right| d r\right)\right\}=: \alpha(t) .
\end{aligned}
$$

Let $b>1$. In view of the $b$-periodicity of the system, this is equal to the value of the set function

$$
\begin{gathered}
M^{-1} \int P^{b}(d \Phi)\left\{\sum _ { i \in I ( \Phi ) } \mathbb { 1 } _ { W _ { b } } ( x _ { i } ) \left[\sum_{y \in \mathbb{Z}^{d}} \mathbb{1}_{B+b y}\left(x_{i}(t)\right)\right.\right. \\
\left.\left.\cdot\left(\left|v_{i}\right|+\int_{0}^{T} \sum_{j \in I(\Phi)}\left|\nabla U\left(x_{j}(r)-x_{i}(r)\right)\right| d r\right)\right]\right\}=: \lambda(B)
\end{gathered}
$$


at the argument $B=W_{1}$. Here $B$ runs through the $\sigma$-algebra of all Borel subsets of $W_{b}$. It is easy to see that $\lambda$ is a measure on $W_{b}$. Moreover, from the translation invariance and $b$-periodicity of $P^{b}$ it follows that $\lambda$ is translation invariant, too. Of course, this translation invariance is meant modulo $b$. (To understand that consider the torus system by means of which we defined the motion group $S_{t}^{b}$.) Hence $\lambda$ must be a multiple of the Lebesgue measure on $W_{b}$, and so we can continue the above chain by

$$
=b^{-d} M^{-1} \int P^{b}(d \Phi)\left\{\sum_{i \in I(\Phi)} \mathbb{1}_{W_{b}}\left(x_{i}\right)\left(\left|v_{i}\right|+\int_{0}^{T} \sum_{j \in I(\Phi)}\left|\nabla U\left(x_{j}(r)-x_{i}(r)\right)\right| d r\right)\right\} .
$$

Hence $\alpha(t)$ does not depend on $t$ and we get for $t=0$,

$$
=M^{-1} \int P^{b}(d \Phi)\left\{\sum_{i \in I(\Phi)} \mathbb{1}_{W_{1}}\left(x_{i}\right)\left(\left|v_{i}\right|+\int_{0}^{T} \sum_{j \in I(\Phi)}\left|\nabla U\left(x_{j}(r)-x_{i}(r)\right)\right| d r\right)\right\} .
$$

Using the estimate $|v| \leqq 1+|v|^{2}$, we get

$$
\begin{aligned}
\leqq & M^{-1}\left(\bar{m}+\int P^{b}(d \Phi)\left[2 e\left(\Phi, W_{1}\right)-\sum_{i \neq j} \mathbb{1}_{W_{1}}\left(x_{i}\right) U\left(x_{j}-x_{i}\right)\right.\right. \\
& \left.\left.+\sum_{i, j} \mathbb{1}_{W_{1}}\left(x_{i}\right) \int_{0}^{T}\left|\nabla U\left(x_{j}(r)-x_{i}(r)\right)\right| d r\right]\right) \\
\leqq & M^{-1}\left(\bar{m}+2 \bar{e}+(1+2 R)^{2 d} \bar{u} \bar{q}^{b}\right. \\
& \left.+\int_{0}^{T}\left[\int P^{b}(d \Phi) \sum_{i, j} \mathbb{1}_{W_{1}}\left(x_{i}\right)\left|\nabla U\left(x_{j}(r)-x_{i}(r)\right)\right|\right] d r\right) .
\end{aligned}
$$

Let us denote the expression

$$
\int P^{b}(d \Phi) \sum_{i, j \in I(\Phi)} \mathbb{1}_{W_{1}}\left(x_{i}(t)\right)\left|\nabla U\left(x_{j}(r)-x_{i}(r)\right)\right|
$$

by $\beta(t)$. The same arguments as above yield the independence of $\beta$ from $t$. So we may continue the chain of inequalities by

$$
\begin{aligned}
= & M^{-1}\left(\bar{m}+2 \bar{e}+(1+2 R)^{2 d} \bar{u} \bar{q}^{b}\right. \\
& \left.+\int_{0}^{T}\left[\int P^{b}(d \Phi) \sum_{i, j} \mathbb{1}_{W_{1}}\left(x_{i}(r)\right)\left|\nabla U\left(x_{j}(r)-x_{i}(r)\right)\right|\right] d r\right) \\
\leqq & M^{-1}\left[\bar{m}+2 \bar{e}+(1+2 R)^{2 d}\left(\bar{u} \bar{q}^{b}+\overline{\bar{u}} \int_{0}^{T} \bar{q}_{r}^{b} d r\right)\right] .
\end{aligned}
$$

Finally, by (2.9) this can be estimated by

$$
\leqq M^{-1}\left[\bar{m}+2 \bar{e}+(1+2 R)^{2 d}(\bar{u}+T \bar{u}) C_{0}\right],
$$

where $C_{0}$ does not depend on $b$. Altogether we have shown

Lemma 6. There is some constant $k_{1}$ (not depending on $b$ or $t$ ) such that

$$
P^{b}\left(Y_{t, T}^{M, b}\right) \leqq k_{1} T M^{-1}, \quad T, b \geqq 1 .
$$


That means that the maximum velocity of a particle chosen from a bounded region (at any given time $t$ ) remains statistically bounded for finite intervals of time uniformly in the periodicity constant $b$.

Remark. Of course, instead of using the unit cube in the definition of $Y_{t, T}^{M, b}$ we could have taken any bounded Borel set $B$. If we denote the set defined in that manner by $Y_{t, T, B}^{M, b}$, we get

$$
P^{b}\left(Y_{t, T, B}^{M, b}\right) \leqq k_{1}(B) T M^{-1}, \quad T, b \geqq 1 .
$$

\section{Trajectory Processes}

Let $\mathscr{C}$ be the set of all continuous $\mathbb{R}^{d}$-valued functions $\xi$ defined on $[0, \infty)$ which have the property that $\lim _{t \rightarrow \infty} e^{-t}|\xi(t)|=0$. We equip this set with the following metric:

$$
\varrho_{\mathscr{C}}(\xi, \eta):=\sup _{t \geqq 0} e^{-t}|\xi(t)-\eta(t)|, \quad \xi, \eta \in \mathscr{C} .
$$

It is easy to see that $\mathscr{C}$ is a complete separable metric space. Consider the set $\mathscr{S}=\mathbb{R}^{d} \times \mathbb{R}^{d} \times \mathscr{C}$ endowed with the metric $\varrho_{\mathscr{S}}:=d+\varrho_{\mathscr{C}} /\left(1+\varrho_{\mathscr{C}}\right)$. This makes $\mathscr{S}$ a complete separable metric space, too. Hence we may consider $\mathscr{S}$ as phase space in the sense of point field theory (cf. [10, Chap. 4]). Here $\mathscr{C}$ plays the role of an additional "mark space." Let $[\mathscr{M}, \mathbf{M}]$ denote the corresponding space of counting measures, with the canonical $\sigma$-field of Borel sets (with respect to the vague topology) $\mathbf{M}$. The set of all probability measures on $[\mathscr{M}, \mathbf{M}]$ we equip with the weak topology [10, Chap. 4].

Let us consider the trajectory $x_{i}$ of a particle $\left[x_{i}, v_{i}\right]$ belonging to some $\Phi \in \mathrm{M}_{b}$. Now consider the relative (or displacement-) trajectory $\zeta_{i}:=x_{i}-x_{i}$, and add it as a "mark" to $\left[x_{i}, v_{i}\right]$, getting $\left[x_{i}, v_{i}, \zeta_{i}\right] \in \mathscr{S}$, where the fact that $\zeta_{i} \in \mathscr{C}$ easily follows from the boundedness of velocities in the periodic case. In that way we get a family of mappings

$$
F^{b}: \mathrm{M}_{b} \curvearrowright \mathscr{M}, \quad b \geqq 1 .
$$

We have

Lemma 7. For each $b \geqq 1$ the mapping $F^{b}$ is continuous with respect to the vague topologies in $\mathrm{M}_{b}$ and $\mathscr{M}$.

Proof. The vague topology is metrizable [10, Chap. 1.9]. So, let $\left\{\Phi_{\ell}\right\}$ be a sequence of initial conditions from $\mathrm{M}_{b}$ such that $\Phi_{\ell} \rightarrow \Phi \in \mathrm{M}_{b}$ vaguely. In view of the criterion for vague convergence given in Chap. 1 , this is equivalent to the assertion that for some $\ell_{0}$ we have

$$
\Phi_{\ell}^{\times}\left(W_{b}\right)=\Phi^{\times}\left(W_{b}\right)=: n, \quad \ell \geqq \ell_{0},
$$

and that the element $a_{\ell} \in \mathbb{T}^{n d} \times \mathbb{R}^{n d}$ corresponding to $\Phi_{\ell}, \ell \geqq \ell_{0}$, converges towards $a$ (the element corresponding to $\Phi$ ). Now again from the theorem on the continuous dependence of the solutions of ordinary differential equations from the initial values we infer that, for any bounded interval of time $[0, T]$, the trajectory 
$a_{\ell}(t)$ converges uniformly in $t \in[0, T]$ towards $a(t)$, and so do the "unrolled" trajectories of the particles of $\Phi_{\ell}$, such that, for any particle $\left[x_{i}, v_{i}\right]$ in $\Phi$ and the corresponding particles $\left[x_{i, \ell}, v_{i, \ell}\right]$ in $\Phi_{\ell}$ we have

$$
\zeta_{i, \ell}(t)=x_{i, \ell}(t)-x_{i, \ell} \underset{\ell \rightarrow \infty}{\longrightarrow} \zeta_{i}(t)=x_{i}(t)-x_{i}
$$

(uniformly in $t$ for bounded $t$ ). On the other hand from $a_{\ell} \rightarrow a$ we infer that for the energy (being a continuous function on $\mathbb{T}^{n d} \times \mathbb{R}^{n d}$ ) we have $e\left(a_{\ell}\right) \rightarrow e(a)$. So all $e\left(a_{\ell}\right)$ are bounded by some $E_{0}$, such that

$$
\left|\dot{\zeta}_{i}(t)\right|,\left|\dot{\zeta}_{i, \ell}(t)\right| \leqq \sqrt{2 E_{0}+n^{2} \bar{u}}=: K_{0}
$$

for $t \geqq 0$. So we have for any $T>1$

$$
\begin{aligned}
& \limsup _{\ell \rightarrow \infty} \varrho_{\mathscr{C}}\left(\zeta_{i, \ell}, \zeta_{i}\right) \leqq \limsup _{\ell \rightarrow \infty} \sup _{0 \leqq t \leqq T} e^{-t}\left|\zeta_{i, \ell}(t)-\zeta_{i}(t)\right| \\
& \quad+\sup _{T<t} 2 e^{-t} K_{0} t=0+2 K_{0} T e^{-T} \underset{T \rightarrow \infty}{\longrightarrow} 0
\end{aligned}
$$

such that $\varrho_{\mathscr{C}}\left(\zeta_{i, \ell}, \zeta_{i}\right) \rightarrow 0$ as $\ell \rightarrow \infty$.

In view of the vague convergence criterion given in Chap. 1, we may conclude that $F^{b}\left(\Phi_{\ell}\right) \rightarrow F^{b}(\Phi)$.

So any point field distribution $P$ generates a family of probability measures $\mathscr{P}^{b}$ on $\mathscr{M}$ defined by

$$
\mathscr{P}^{b}:=P^{b} \circ\left(F^{b}\right)^{-1} .
$$

Suppose that $P$ fulfills the assumptions given above, i.e. $P$ is translation invariant and has a finite specific energy and mean particle density. Now we can prove the following:

Proposition 1. The family $\left\{\mathscr{P}^{b}\right\}$ is relatively compact with respect to the weak topology.

Proof. 1. Using the compactness criteria 4.1.5 and 4.1.3 in [10], we see that the proposition would be a consequence of the following assertion: For any closed bounded subset $X$ of $\mathscr{S}$ and any $\varepsilon>0$ there is a compact $D_{X, \varepsilon} \subset X$ such that

$$
\sup _{b>1} \int \mathscr{P}^{b}(d \varphi) \varphi\left(X \backslash D_{X, \varepsilon}\right)<\varepsilon .
$$

We shall show this in the sequel. Without any loss of generality we may choose $X$ as

$$
X:=\{[x, v, \zeta] \in \mathscr{S}:|x| \leqq K\} .
$$

We introduce the following sets (depending on a constant $L$ )

$$
\begin{aligned}
& X_{L}:=\left\{[x, v, \zeta] \in \mathscr{S}:|x| \leqq K,|v| \leqq L,\left|\zeta(s)-\zeta\left(s^{\prime}\right)\right|\right. \\
& \leqq\left|s-s^{\prime}\right| L(1+t)^{-1} e^{t} \text { for all } s, s^{\prime}, t \geqq 0 \\
&\text { with } \left.s \leqq t, s^{\prime} \leqq t \text { and } \zeta(0)=0\right\} .
\end{aligned}
$$

Clearly the $X_{L}$ are closed subsets of $\mathscr{S}$. Fix some $L$ and consider any sequence $\left\{s_{i}\right\}_{i \in \mathbb{N}}=\left\{\left[x_{i}, v_{i}, \zeta_{i}\right]\right\}_{i \in \mathbb{N}}$ of elements of $X_{L}$. For each natural number $N$ we find a 
subsequence $\left\{s_{i^{\prime}}\right\}$ of $\left\{s_{i}\right\}$ such that the $x_{i^{\prime}}$ and $v_{i^{\prime}}$ converge towards some $x$ and $v$ and such that $\zeta_{i}$, converges uniformly on the interval $[0, N]$ towards a continuous function defined on $[0, N]$. This is a simple consequence of the Arzelà-Ascoli theorem. By the diagonal method we find a subsequence $\left\{\zeta_{i^{\prime \prime}}\right\}$ of $\left\{\zeta_{i^{\prime}}\right\}$ converging pointwise towards a continuous function $\zeta$ defined on $[0, \infty)$ such that the convergence is uniform on each bounded interval of time.

Obviously for each $i$ we have

$$
\left|\zeta_{i}(t)\right| \leqq L \int_{0}^{t}(1+s)^{-1} e^{s} d s, \quad t \geqq 0
$$

such that the same estimate holds for $\zeta$. Since $\int_{0}^{t}(1+s)^{-1} e^{s} d s=o\left(e^{t}\right), \zeta$ is an element of $\mathscr{C}$. We have, for any $N>0$ :

$$
\begin{aligned}
& \limsup _{i^{\prime \prime} \rightarrow \infty} \varrho_{\mathscr{C}}\left(\zeta_{i^{\prime \prime}}, \zeta\right)=\limsup _{i^{\prime \prime} \rightarrow \infty} \sup _{t \geqq 0} e^{-t}\left|\zeta_{i^{\prime \prime}}(t)-\zeta(t)\right| \\
& \leqq \\
& \liminf _{N \rightarrow \infty}\left[\limsup _{i^{\prime \prime} \rightarrow \infty} \sup _{0 \leqq t \leqq N} e^{-t}\left|\zeta_{i^{\prime \prime}}(t)-\zeta(t)\right|\right. \\
& \left.\quad+\limsup _{i^{\prime \prime} \rightarrow \infty} \sup _{t>N} e^{-t}\left(\left|\zeta_{i^{\prime \prime}}(t)\right|+|\zeta(t)|\right)\right] \\
& \leqq 0+\liminf _{N \rightarrow \infty} 2 L \sup _{t>N} e^{-t} \int_{0}^{t}(1+s)^{-1} e^{s} d s=0 .
\end{aligned}
$$

Hence we have $s_{i^{\prime \prime}} \underset{\varrho_{\mathscr{G}}}{\longrightarrow}[x, v, \zeta] \in X_{L}$ as $i^{\prime \prime} \rightarrow \infty$, such that $X_{L}$ is compact.

2. With the help of (2.9), Lemma 6 and the Schwarz inequality, we get

$$
\begin{aligned}
\int \mathscr{P}^{b}(d \varphi) \varphi\left(X \backslash X_{L}\right)= & \int \mathscr{P}^{b}(d \varphi)\left[\varphi\left(X \backslash X_{L}\right) \mathbb{1}_{\left\{\psi \in \mathscr{M}: \psi\left(X \backslash X_{L}\right)>0\right\}}(\varphi)\right] \\
\leqq & \left(\int \mathscr{P}^{b}(d \varphi)\left[\varphi\left(X \backslash X_{L}\right)\right]^{2}\right)^{1 / 2}\left(\mathscr{P}^{b}\left(\varphi\left(X \backslash X_{L}\right)>0\right)\right)^{1 / 2} \\
\leqq & \left(\int P^{b}(d \Phi)\left[\Phi^{\times}\left(B_{K}\right)\right]^{2}\right)^{1 / 2} \\
& \cdot\left(P ^ { b } \left(\left\{\Phi \in \mathrm{M}_{b}: \text { There is a }\left[x_{i}, v_{i}\right] \text { in } \Phi\right.\right.\right. \text { such that } \\
& \left.\left.\left.x_{i} \in B_{K} \text { and } \sup _{t \geqq 0}(1+t) e^{-t}\left|\dot{x}_{i}(t)\right|>L\right\}\right)\right)^{1 / 2} \\
\leqq & (2 K)^{d}\left(\bar{q}^{b}\right)^{1 / 2}(2 K)^{d / 2}\left(\sum_{j=0}^{\infty} P^{b}\left(Y_{0, j+1}^{(1+j)^{-1} e^{j} L, b}\right)\right)^{1 / 2} \\
\leqq & c\left(\sum_{j=0}^{\infty}(j+1)^{2} e^{-j}\right)^{1 / 2} L^{-1 / 2},
\end{aligned}
$$

where $c$ is some constant not depending on $b$. Hence we have $\lim _{L \rightarrow \infty}\left(\sup _{b>1} \int \mathscr{P}^{b}(d \varphi) \varphi\left(X \backslash X_{L}\right)\right)=0$.

\section{The Existence Theorem}

The proposition proved above yields the key to show the existence of Newtonian dynamics.

Theorem. Let the potential function $U$ fulfill the assumptions given in the introduction. Let $P$ be the probability measure of a translation invariant particle 
system (with phase space $\mathbb{R}^{d} \times \mathbb{R}^{d}$ ) which is supposed to possess finite particle density and specific energy. Then for P-almost all configurations $\Phi_{0}$ there exists a solution to $(N)$ with initial condition $\Phi_{0}$.

Proof. 1. The results above yield a family of probability measures $\left\{\mathscr{P}^{b}\right\}_{b>1}$ on $[\mathscr{M}, \mathbf{M}]$. From Proposition 1 we derive that there is a limit point of this family, so that for some $\mathscr{P}$ and a certain sequence $b_{i} \uparrow \infty$, we have $\mathscr{P}^{b_{2}} \underset{i \rightarrow \infty}{\Longrightarrow} \mathscr{P}$.

We introduce the following subsets of $\mathscr{M}$ :

$$
\begin{aligned}
Y_{1}:=\{\varphi \in \mathscr{M}: & \text { For each } i \in I(\varphi) \text { with } x_{i} \in \dot{B}_{1}, \\
& \text { we have } \zeta_{i}(t)=v_{i} t-\int_{0}^{t} d s \int_{0}^{s} d r \sum_{j \in I(\varphi)} \nabla U\left(x_{i}-x_{j}+\zeta_{i}(r)-\zeta_{j}(r)\right) \\
& \text { for } 0 \leqq t \leqq 1\},
\end{aligned}
$$

$Y_{2}^{M}:=\left\{\varphi \in \mathscr{M}:\right.$ For each $i \in I(\varphi)$ with $x_{i} \in \stackrel{\circ}{B}_{1}$, we have

$$
\left.\sup _{0 \leqq s \leqq 1}\left|\zeta_{i}(s)\right| \leqq M\right\}
$$

$Y_{3}^{M, N}:=\{\varphi \in \mathscr{M}:$ For each $i \in I(\varphi)$ such that there is a

$$
\tau \in[0,1] \text { with } x_{i}+\zeta_{i}(\tau) \in \stackrel{\circ}{B}_{R+M+1} \text {, }
$$

we have $\left.\left|x_{i}\right| \leqq N\right\}$.

Here $M$ and $N$ are natural numbers with $M, N>1$. We set

$$
Y^{M, N}:=Y_{1} \cap Y_{2}^{M} \cap Y_{3}^{M, N} \text {. }
$$

2. $Y^{M, N}$ is a vaguely closed set. Indeed, let $\left\{\varphi_{\ell}\right\}_{\ell=1,2, . .}$ be a sequence of elements of $Y^{M, N}$ tending to some $\varphi \in \mathscr{M}$. Let be given representations

$$
\begin{aligned}
\varphi & =\sum_{1 \leqq i<I} \delta_{\left[x_{i}, v_{i}, \zeta_{i}\right]}, \\
\varphi_{\ell} & =\sum_{1 \leqq i<N(\ell)} \delta_{\left[x_{i, \ell}, v_{i, \ell}, \zeta_{i, \ell]}\right]}+\psi_{\ell},
\end{aligned}
$$

such that the properties Ai)-Aiii) (introduced in Chap. 1) are fulfilled (in the context of our special phase space $\mathscr{S}$ ). Now suppose that $x_{1} \in \dot{B}_{1}$. Hence for almost all $\ell$ we have $x_{1, \ell} \in \stackrel{\circ}{B}_{1}$. Since all $\varphi_{\ell} \in Y_{2}^{M}$, we have $\sup _{0 \leqq s \leqq 1}\left|\zeta_{1, \ell}(s)\right| \leqq M$ for almost all $\ell$, such that $\sup _{0 \leqq s \leqq 1}\left|\zeta_{1}(s)\right| \leqq M$, too. So $\varphi \in Y_{2}^{M}$ such that $Y_{2}^{M}$ is a closed set.

Hence within the time interval $[0,1]$ the vector $x_{1}+\zeta_{1}(s)$ does not leave the set $B_{M+1}$. Let $J$ denote the set of all indices such that $x_{i}+\zeta_{i}\left(\tau_{i}\right) \in \dot{B}_{M+R+1}$ for some $\tau_{i} \in[0,1]$.

For any $i \in J$ we have $x_{i, \ell}+\zeta_{i, \ell}\left(\tau_{i}\right) \in \dot{B}_{M+R+1}$ for almost all $\ell$, such that those $[i, \ell]$ fulfill $\left|x_{i, \ell}\right| \leqq N$, from which we deduce that $\left|x_{i}\right| \leqq N$. So $\varphi \in Y_{3}^{M, N}$, and this is a closed set, too. Since the closed set $\mathscr{S}_{N}:=\{[x, v, \zeta] \in \mathscr{S}:|x| \leqq N\}$ is $\varrho_{\mathscr{S}}$-bounded, we may deduce from the fact that $\varphi$ is a (locally finite) counting measure, that $J$ is a finite set, contained in the finite set $J^{\prime}$ of all indices $i$ with $s_{i}:=\left[x_{i}, v_{i}, \zeta_{i}\right] \in \mathscr{S}_{N}$. Now, since $\mathscr{S}_{N}$ is closed and bounded, in accordance with Aii) we find a $\ell_{0}$ such that for 
$\ell \geqq \ell_{0}$ we have

$$
\psi_{\ell}\left(\mathscr{S}_{N}\right)=0, \quad s_{i, \ell}:=\left[x_{i, \ell}, v_{i, \ell}, \zeta_{i, \ell}\right] \notin \mathscr{S}_{N} \text { for } i \notin J^{\prime} .
$$

Hence for each $\ell \geqq \ell_{0}$,

$$
\zeta_{1, \ell}(t)=v_{1, \ell} t-\int_{0}^{t} d s \int_{0}^{s} d r \sum_{i \in J^{\prime}} \nabla U\left(x_{1, \ell}-x_{i, \ell}+\zeta_{1, \ell}(r)-\zeta_{i, \ell}(r)\right), \quad 0 \leqq t \leqq 1 .
$$

Since, as we have shown, $\varphi \in Y_{3}^{M, N}$, no trajectory $x_{i}+\zeta_{i}$ with $i \notin J^{\prime}$ for $t \in[0,1]$ hits $\dot{B}_{M+R+1}$, hence any such trajectory fulfills $\left|x_{1}-x_{i}+\zeta_{1}(t)-\zeta_{i}(t)\right| \geqq R$ for $0 \leqq t \leqq 1$. From (6.1) we get

$$
\zeta_{1}(t)=v_{1} t-\int_{0}^{t} d s \int_{0}^{s} d r \sum_{1 \leqq i<I} \nabla U\left(x_{1}-x_{i}+\zeta_{1}(r)-\zeta_{i}(r)\right), \quad 0 \leqq t \leqq 1 .
$$

We derived $\varphi \in Y_{1}$, such that $\varphi \in Y^{M, N}$. This proves the fact that $Y^{M, N}$ is a closed set.

3. Therefore we have (cf. [11, Theorem 2.1])

$$
\mathscr{P}\left(Y^{M, N}\right) \geqq \limsup _{i \rightarrow \infty} \mathscr{P}^{b_{i}}\left(Y^{M, N}\right) .
$$

We can estimate

$$
\mathscr{P}^{b}\left(Y^{M, N}\right) \geqq 1-\left[\mathscr{P}^{b}\left(\mathscr{M}-Y_{1}\right)+\mathscr{P}^{b}\left(\mathscr{M}-Y_{2}^{M}\right)+\mathscr{P}^{b}\left(\mathscr{M} \backslash Y_{3}^{M, N}\right)\right] .
$$

Clearly,

$$
\mathscr{P}^{b}\left(\mathscr{M} \backslash Y_{1}\right)=0
$$

On the other hand using Lemma 6 we get

$$
\begin{aligned}
\sup _{b>1} \mathscr{P}^{b}\left(\mathscr{M} \backslash Y_{2}^{M}\right) & \leqq \sup _{b>1} \mathscr{P}^{b}\left(\left\{\varphi \in \mathscr{M}: \text { There is an } i \in I(\varphi) \text { with } x_{i} \in \mathscr{W}_{2}\right.\right. \\
& \text { and }|\zeta(t)|>M \text { for some } t \in[0,1]\}) \\
& \leqq 2^{d} \sup _{b>1} P^{b}\left(Y_{0,1}^{M, b}\right) \leqq 2^{d} k_{1} M^{-1} \underset{M \rightarrow \infty}{\longrightarrow} 0 .
\end{aligned}
$$

4. For real numbers $M, N>1$ and natural numbers $L$ we introduce the following sets:

$$
\begin{aligned}
X_{b}^{M, N, L}:=\left\{\Phi \in \mathrm{M}_{b}:\right. & \text { There is an } i \in I\left(F^{b}(\Phi)\right) \text { and a } \\
& \text { natural number } A \leqq L \text { such that } \\
& \left.x_{i}+\zeta_{i}\left(\frac{A}{L}\right) \in B_{M+R+2}^{\circ} \text { and }\left|x_{i}\right|>N\right\} .
\end{aligned}
$$

Now we obtain for any natural number $L$

$$
\left(F^{b}\right)^{-1}\left(\mathscr{M} \backslash Y_{3}^{M, N}\right) \cong X_{b}^{M, N, L} \cup Z_{b}^{M, L},
$$

where

$$
\begin{aligned}
Z_{b}^{M, L}:=\left\{\Phi \in \mathrm{M}_{b}:\right. & \text { There is an } i \in I(\Phi) \text { such that } \\
& x_{i}+\zeta_{i}(\tau) \in \dot{B}_{M+R+2} \text { and }\left|\dot{\zeta}_{i}(\tau)\right| \geqq 2 L \\
& \text { for some } 0 \leqq \tau \leqq 1\} .
\end{aligned}
$$


So we have for any fixed $M$ [with the help of Lemma 5 and (4.1)] $\limsup _{N \rightarrow \infty} \sup _{b>1} \mathscr{P}^{b}\left(\mathscr{M} \backslash Y_{3}^{M, N}\right)$

$\leqq \limsup _{L \rightarrow \infty} \limsup _{N \rightarrow \infty} \sup _{b>1} P^{b}\left(X_{b}^{M, N, L}\right)+\limsup _{L \rightarrow \infty} \sup _{b>1} P^{b}\left(Z_{b}^{M, L}\right)$

$\leqq \limsup _{L \rightarrow \infty} \limsup _{N \rightarrow \infty} \sup _{b>1} \sum_{A=0}^{L} P^{b}\left(\left\{\Phi \in \mathrm{M}_{b}\right.\right.$ : There is an $i \in I(\Phi)$

$$
\begin{aligned}
& \text { with } x_{i}+\zeta_{i}\left(\frac{A}{L}\right) \in \stackrel{\circ}{B}_{M+R+2} \\
& \text { and }\left|\dot{\zeta}_{i}(\tau)\right|>N-(M+R+2) \\
& \text { for some } \tau \in[0,1]\}) \\
& +\limsup \sup _{L \rightarrow \infty} P_{b>1}^{b}\left(\left\{\Phi \in \mathrm{M}_{b}: \sup _{0 \leqq \tau \leqq 1} e_{0}\left(S_{\tau}^{b}(\Phi), \stackrel{\circ}{B}_{M+R+2}\right) \geqq 2 L^{2}\right\}\right) \\
& \leqq \limsup _{L \rightarrow \infty} \limsup _{N \rightarrow \infty} \sup _{b>1} \sum_{A=0}^{L} P^{b}\left(Y_{A / L, 1, B_{M+R+2}}^{N-(M+R+2), b}\right)+0 \\
& \leqq \limsup _{L \rightarrow \infty} \limsup _{N \rightarrow \infty}(L+1) k_{1}\left(\stackrel{\circ}{B}_{M+R+2}\right)(N-M-R-2)^{-1}=0 \text {. }
\end{aligned}
$$

5. Altogether we have, using (6.2)

$$
\lim _{M \rightarrow \infty} \liminf _{N \rightarrow \infty} \mathscr{P}\left(Y^{M, N}\right)=1
$$

Let us denote by $Y_{3}$ the measurable set $\bigcap_{M} \bigcup_{N} Y_{3}^{M, N}$. Obviously

$$
\begin{aligned}
Y_{3}=\{\varphi \in \mathscr{M}: & \text { For each bounded } B \in \mathbb{R}^{d} \text { there is just a finite number } \\
& \text { of } i \in I(\varphi) \text { such that } x_{i}+\zeta_{i}(\tau) \in B \\
& \text { for some } \tau \in[0,1]\} .
\end{aligned}
$$

From (6.3) and the definition of $Y^{M, N}$ we get $\mathscr{P}\left(Y_{3}\right)=1$ such that, setting

$$
Y^{M}:=Y_{1} \cap Y_{2}^{M} \cap Y_{3}=\left[\bigcup_{N}\left(Y_{1} \cap Y_{2}^{M} \cap Y_{3}^{M, N}\right)\right] \cap Y_{3},
$$

we get

$$
\lim _{M \rightarrow \infty} \mathscr{P}\left(Y^{M}\right)=\mathscr{P}\left(Y_{1} \cap Y_{3}\right)=1 .
$$

Since the restriction to the finite time interval $[0,1]$ and the unit ball $B_{1}$ was arbitrary, we see that

$$
\begin{aligned}
\mathscr{P}(\{\varphi \in \mathscr{M}: & \text { For each bounded } B \in R^{d} \text { and each } T>0 \text { there is } \\
& \text { just a finite number of } i \in I(\varphi) \text { such that } \\
& x_{i}+\zeta_{i}(\tau) \in B \text { for some } \tau \leqq T \text {, and for each } i \in I(\varphi) \\
& \text { we have } \\
& \zeta_{i}(t)=v_{i} t-\int_{0}^{t} d s \int_{0}^{s} d r \sum_{j \in \Pi(\varphi)} \nabla U\left(x_{i}-x_{j}+\zeta_{i}(r)-\zeta_{j}(r)\right) \\
& \text { for } t \geqq 0\})=1 .
\end{aligned}
$$


We denote the (measurable) event considered above by $Y_{0}$. Then, of course, any $\varphi \in Y_{0}$ yields a solution to $(N)$ : The collection of $\mathbb{R}^{d}$-valued functions $x_{i}+\zeta_{i}$ resulting from the atoms $\left[x_{i}, v_{i}, \zeta_{i}\right]$ of $\varphi$ fulfils $(N)$ with initial condition given by the atoms of $\Pi \varphi$, where $\Pi$ is the projector defined by

$$
\Pi: \varphi \in \mathscr{M} \curvearrowright \Pi_{\varrho}=\sum_{i \in I(\varphi)} \delta_{\left[x_{i}, v_{i}\right]} \in \mathrm{M} .
$$

6. Obviously $\Pi$ is a continuous mapping from $\mathscr{M}$ to $\mathrm{M}$. We have in view of the continuity Theorem 5.1 in [11] and Lemma 3 ,

$$
\mathscr{P} \circ \Pi^{-1}=\lim _{i \rightarrow \infty} \mathscr{P}^{b_{i}} \circ \Pi^{-1}=\lim _{i \rightarrow \infty} P^{b_{i}}=P,
$$

since

$$
\Pi \circ F^{b}=\mathrm{id}_{\mathrm{M}_{b}} .
$$

As mentioned above, $\left(\mathscr{S}, \varrho_{\mathscr{S}}\right)$ is a complete separable metric space. Hence (cf. [10, Chap. 4]), $[\mathscr{M}, \mathbf{M}]$ is a standard Borel space. So we may form regular conditional probabilities $\mathscr{P}\left(* \mid \mathbf{M}_{\Pi}\right)$, where $\mathbf{M}_{\Pi}:=\Pi^{-1}(\mathfrak{M})$ is the (countably generated) $\sigma$-subfield of $\mathbf{M}$ generated by $\Pi$. We have $\mathscr{P}\left(Y_{0} \mid \mathbf{M}_{\Pi}\right)(\varphi)=1$ for $\mathscr{P}$-almost all $\varphi \in \mathscr{M}$. So in view of the relation $\mathscr{P} \circ \Pi^{-1}=P$ and of the $\mathbf{M}_{\Pi}$-measurability of $\mathscr{P}\left(Y_{0} \mid \mathbf{M}_{\Pi}\right)(\varphi)$, we get that for $P$-almost all $\Phi_{0} \in \mathrm{M}$ and all $\varphi \in \Pi^{-1}\left(\Phi_{0}\right)$, the relation $P\left(Y_{0} \mid \mathbf{M}_{\Pi}\right)(\varphi)=1$ holds. On the other hand, for $P$-almost all $\Phi_{0} \in \mathrm{M}$ and any $\varphi \in \Pi^{-1}\left(\Phi_{0}\right)$, we have $\mathscr{P}\left(\Pi^{-1}\left(\Phi_{0}\right) \mid \mathbf{M}_{\Pi}\right)(\varphi)=1$ (see, for instance, [13, Theorem 1.1.8]). So for a certain set $\tilde{M}$ in $\mathfrak{M}$ of $P$-measure 1 we have $\Pi^{-1}\left(\Phi_{0}\right) \cap Y_{0} \neq \emptyset$ for $\Phi_{0} \in \tilde{\mathrm{M}}$, such that according to 5 . there is a solution to $(N)$ with initial condition $\Phi_{0}$ for any $\Phi_{0} \in \tilde{\mathrm{M}}$, and $\tilde{\mathrm{M}}$ has $P$-measure 1 .

Acknowledgements. This paper was stimulated during my stay at IPPI, Moscow, in 1983. I am very grateful for the warm hospitality at the Institute for Problems of Information Transmission and want to express my thankfulness especially to R. L. Dobrushin, A. N. Rybko, and Yu. M. Suhov.

\section{References}

1. Lanford III, O.E.: The classical mechanics of one-dimensional systems of infinitely many particles. Commun. Math. Phys. 9, 169-191 (1968)

2. Lanford III, O.E.: Time evolution of large classical systems. In: Dynamical systems, theory, and applications. Lecture Notes in Physics, Vol. 38, pp. 1-111. Berlin, Heidelberg, New York: Springer 1975

3. Dobrushin, R.L., Fritz, J.: Non-equilibrium dynamics of one-dimensional infinite particle systems with a hardcore interaction. Commun. Math. Phys. 55, 275-292 (1977)

4. Fritz, J., Dobrushin, R.L.: Non-equilibrium dynamics of two-dimensional infinite particle systems with a singular interaction. Commun. Math. Phys. 57, 67-81 (1977)

5. Sinai, Ya.G.: Construction of dynamics in one-dimensional systems of statistical mechanics. Theor. Math. Phys. 11, 248-258 (1972)

6. Sinai, Ya.G.: Construction of cluster dynamics for dynamical systems of statistical mechanics. Vestnik Moskovskogo University, 152-158 (1974)

7. Marchioro, C., Pellegrinotti, A., Presutti, E.: Existence of time-evolution of $v$-dimensional statistical mechanics. Commun. Math. Phys. 40, 175-185 (1975)

8. Alexander, R.: Time evolution for infinitely many hard spheres. Commun. Math. Phys. 49, 217-232 (1976) 
9. Fritz, J.: Some remarks on non-equilibrium dynamics of infinite particle systems. Preprint 1983 (to appear in J. Math. Phys.)

10. Kerstan, J., Matthes, K., Mecke, J.: Infinitely divisible point processes. Moscow: Nauka 1982 (Russian edition)

11. Billingsley, P.: Convergence of probability measures. New York, London, Sydney, Toronto: Wiley 1969

12. Arnold, V.I.: Gewöhnliche Differentialgleichungen. Berlin: Deutscher Verlag der Wissenschaften 1979

13. Strook, D.W., Varadhan, S.R.S.: Multidimensional diffusion process. Berlin, Heidelberg, New York: Springer 1979

14. Siegmund-Schultze, Ra.: Non-equilibrium dynamics of infinite particle systems in the multidimensional translation invariant case (existence). Preprint. Inst. Math. 1984

15. Gurevich, B.M., Sukhov, Yu.M.: Time evolution of Gibbs states in one-dimensional classical statistical mechanics. Doklady Akad. Nauk SSSR 242, 276-279 (1978) (in Russian)

16. Calderoni, P., Caprino, S.: Time evolution of infinitely many particles: An existence theorem. J. Stat. Phys. 28 (4), 815-833 (1982)

Communicated by Ya. G. Sinai

Received August 20, 1984; in revised form January 5, 1985 
